CIENCIA Y SOCIEDAD

Volumen XVIII, Número 1

Enero-Marzo 1993

\author{
DETERMINACION DE ELEMENTOS QUIMICOS \\ EN LAS AGUAS DEL RIO OZAMA \\ MEDIANTE LA TECNICA DE FLUORESCENCIA \\ DE RAYOS-X POR REFLEXION TOTAL (TRXRF)
}

LIC. LETICIA MENDOZA DE CID*

LIC. BELGICA NAÚT M.

\begin{abstract}
Resumen:
Elementos químicos contaminantes fueron cuantificados en las aguas del Río Ozama por la técnica de Fluorescencia de rayos $\mathrm{X}$ por reflexión total, sin utilizar ningún método de proconcentración, con selenio como patrón interno y con una cantidad minima de muestra. Se encontró una alta contaminación química en las aguas del Río Ozama.

Palabras claves: Contaminación, espectrometría de rayos $X$., Río Ozama.
\end{abstract}

\title{
Introducción
}

La fluorescencia de rayos-X es una técnica de análisis que puede clasificarse dentro del campo de la espectroscopía de emisión. Esta técnica, relativamente reciente, es un poderoso instrumento de análisis

* Facultad de Ciencias y Humanidades. 
multielemental simultáneo; es utilizada ampliamente en numerosos campos en los cuales se requiere identificación y cuantificación de elementos químicos. Algunos de sus grandes ventajas son su precisión, exactitud, y su carácter de técnica no destructiva (1).

La reflexión total de rayos-X (TRXRF) es una modificación de la espectrometria o fluorescencia de rayos-X. Esta modificación aumenta sustancialmente los límites de detección ya que se obtiene una reducción drástica del fondo (2).

En el campo de la contaminación ambiental, especificamente en el análisis de aguas, han sido aprovechadas las ventajas que ofrece esta técnica.

Trazas de $\mathrm{V}, \mathrm{Mn}, \mathrm{Fe}, \mathrm{Co}, \mathrm{Ni}, \mathrm{Cu}, \mathrm{Zn}, \mathrm{Mo}, \mathrm{Cd}, \mathrm{Pb}, \mathrm{Y}$ U en aguas del mar del Norte fueron determinadas en el rango de $\mathrm{mg} / \mathrm{kg}$ a $\mathrm{U} / \mathrm{kg}$ usando reflexión total, por Peter Freimann y Diether Schmidt (3).

Andreas Prawge, después de seis meses de trabajos experimentales comprueba que la reflexión total es un método adecuado para determinar trazas de metales en agua de mar con propósito de monitoreo (4). Oscar R. Pérez realizó un estudio del agua potable en la Ciudad de Guatemala con el propósito de determinar el contenido de algunos elementos químicos y verificar si se encuentran en una concentración permisible (2).

\section{Marco Teórico}

El método de reflexión total fue reportado por primera vez en 1971 por Yoneda y Horiuchi (2). Es una modificación de la fluorescencia de rayos-X convencional con la cual se logra una gran reducción del fondo debido a la radiación dispersa y se alcanzan mejores límites de detección con aumento de la sensitividad.

Consiste en hacer incidir el haz de rayos-X sobre la superficie pulida de un reflector de cuarzo en el centro del cual se ha colocado la muestra. El ángulo de incidencia del rayo debe ser sólo de unos pocos minutos de arco para lograr una reflexión total de rayo y que no penetre al porta muestras de cuarzo. De esta manera se elimina casi totalmente la radiación Compton, con lo cual se consigue una drástica reducción del 
fondo del espectro, y la radiación fluorescente que emite la muestra duplica su intensidad.

La reflexión total de un haz de radiaciones ocurre cuando incidente es menor que el ángulo crítico. El ángulo cíftico es el que forma el rayo incidente con la superficie sobre la cual incide (5). Ver gráfica 1.

\section{Gráfica 1}

Análisis Por Fluorescencia de Rayos-X por Reflexión Total

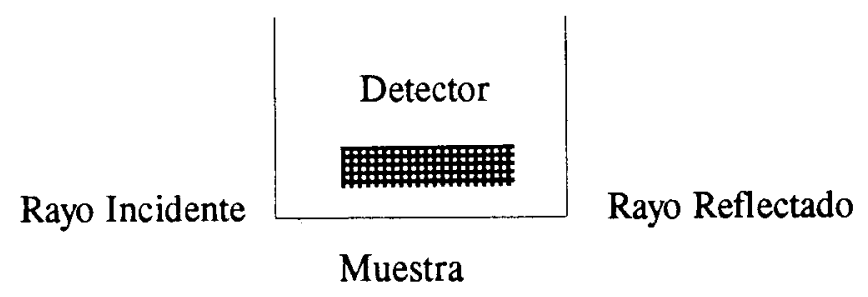

\begin{tabular}{|c|}
\hline Zona de Penetración \\
\hline SUSTRATO DE REFLECTOR \\
\hline
\end{tabular}

El ángulo crítico para la reflexión total depende del material de que está constituido el reflector o porta muestra y de la longitud de onda del rayo incidente:

$$
\begin{aligned}
& \text { Angulo Crítico }=5.4 \times 10^{-10} *(\mathrm{eZ} / \mathrm{A}) \\
& \begin{array}{l}
\mathrm{e}=\text { densidad del material del reflector } \\
\mathrm{Z}=\text { número atómico; } \\
\mathrm{A}=\text { peso atómico y } \\
\quad=\text { longitud de onda del rayo incidente. }
\end{array}
\end{aligned}
$$


El reflector sirve como soporte de la muestra, la cual debe colocarse en el centro de éste como película fina (Thin film). Al mismo tiempo refleja el haz primario de rayo-X.

a - Muestras analizadas para fluorescencia de rayos $\mathrm{X}$.

b - La misma muestra analizada por reflexión total. Nótese la gran diferencia del fondo y el aumento de la intensidad de los picos.

La muestra para la técnica de reflexión total debe ser líquida. La aplicación se hace en el centro del reflector con una micropipeta en volúmenes entre 1-300 microlitros (ver gráfico 1). Luego la muestra se seca con una lámpara o al vacío para que quede una película muy fina (5-8 micrones de diámetro).

La Cuantificación de los elementos presentes en la muestra se realiza en función del elemento que se usa como patrón interno: (2).

$$
\begin{aligned}
& \mathbf{C x}=\mathbf{I}{ }^{*} \mathbf{C p} / \mathbf{K}(\mathbf{x} / \mathbf{p}) * \text { Ip } \\
& \mathbf{C x}=\text { Concentración del elemento a determinar } \\
& \mathrm{Cp}=\text { concentración del patrón } \\
& \mathrm{Ix}=\text { intensidad del elemento a determinar } \\
& \mathrm{Ip}=\text { intensidad del patrón }
\end{aligned}
$$

$\mathrm{K}(\mathrm{x} / \mathrm{p})=$ constante de sensitividad del elemento a determinar respecto del patrón.

\section{Metodología}

Los puntos muestrados fueron los siguientes: No.1 frente a Molinos Dominicanos (próximo a la desembocadura), No. 2 frente a la planta Barceló y Co., No. 3 entre los puentes Mella y Duarte, No. 4 en la confluencia de los ríos Ozama e Isabela. Las muestras fueron tomadas en el centro del río y a $25 \mathrm{~cm}$ de la superficie.

Para la toma y almacenamiento de muestras se utilizaron botellas de polietileno y embudos del mismo material, previamente tratados con HNO al $10 \%$ y por 7 días y enjuagado cuidadosamente con agua destilada, para evitar la contaminación de las muestras con sustancias provenientes de los recipientes (6). 
Cada muestra fue filtrada y los filtros fueron almacenados en bolsas de polietileno y posteriormente sometidos a digestión con $\mathrm{HNO} 3$ concentrado (7).

Tanto las muestras filtradas como los productos de la digestion fueron analizados por separado .

Para analizar las muestras se utilizó un tubo de molibdeno a 20 miliamperes y 40 kilovoltios. Se tomaron $10.0 \mathrm{ml}$ de cada muestra y se agregó a cada una 100 microlitros de selenio $(1000 \mathrm{ppm}$, como patrón interno. Fueron colocados en el reflector 5.0 microl itros de la muestra con el tubo de molibdeno y la emisión de radiación fue recogida por un detector de Germanio hiperpuro. El espectro se obtuvo en un MCA Gamberra serie 35. Los espectros de cada muestra fueron realizados en un microprocesador 80286 utilizando el programa AXIL de la Organización Internacional de Energía Atómica (OIEA).

\section{Resultados y Discusión}

La tabla No. 1 muestra los resultados obtenidos en nuestras mediciones, presentados en los gráficos 2 y 3 .

La muestra tomada en los puntos 1 y 2 arrojó una concentración media de cloro muy elevada. Esta concentración disminuye en los puntos 3 y 4 a medida que nos alejamos a la desembocadura del río. En los puntos 1 y 2 , las elevadas concentraciones de $\mathrm{Cl}$ podrían atribuirse a la proximidad de las aguas del mar, las cuales se mezclan con la del río un largo trecho corriente arriba. En el punto 3, la concentración promedio de $\mathrm{Cl}$ se reduce a una tercera parte de la del punto 2 ; en el punto 5 se reduce a una décima parte aproximadamente, lo cual ratifica lo dicho anteriormente acerca del agua del mar.

En los cuatro puntos analizados las concentraciones de Cl están muy por encima del valor límite para aguas superficiales no Contaminadas el cual es $10 \mathrm{Ug} / \mathrm{l}(8)$.

Los altos niveles del $\mathrm{Cl}$ en las aguas analizadas podrían ser consecuencia de descargas de desechos industriales proveniente de industrias en las cuales se utilizan hidrocarburos clorados como solventes de 


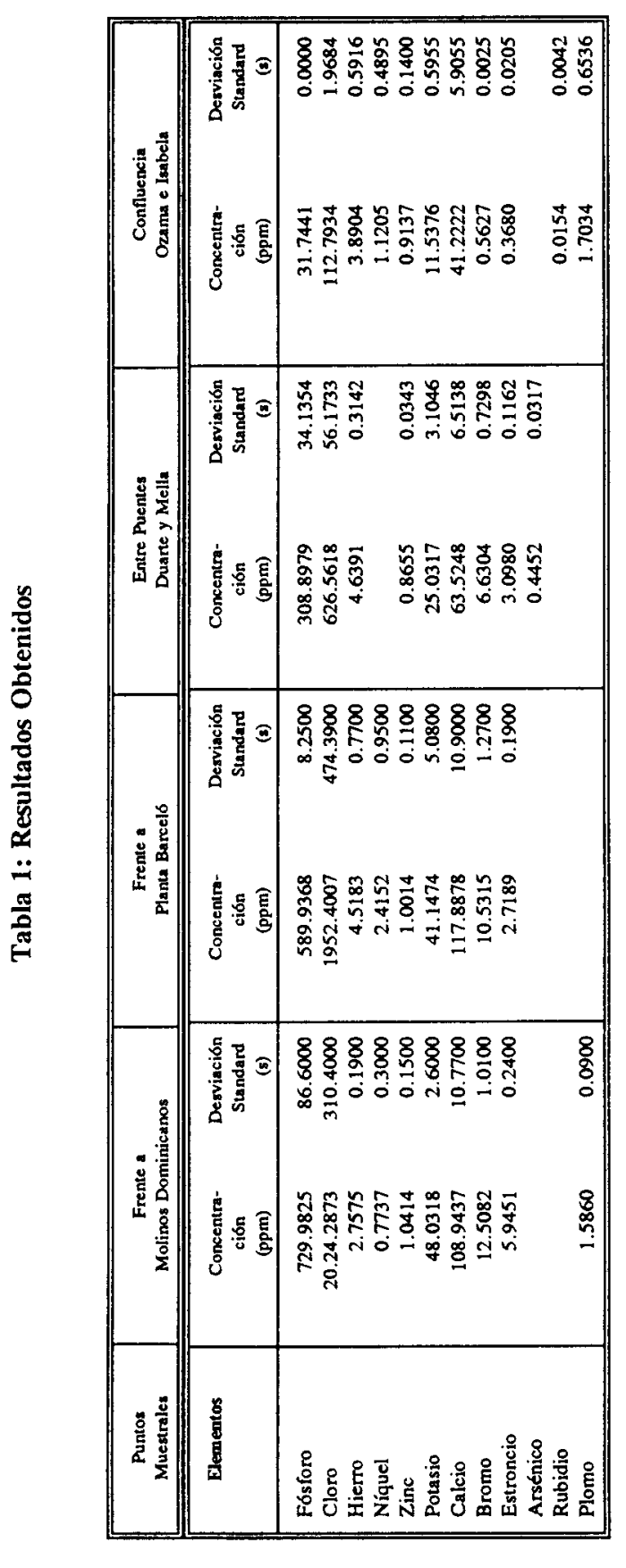


grasas, en la fabricación de envolturas plásticas, en síntesis química y para lavado en seco. Asimismo, la presencia de $\mathrm{Cl}$ en altas concentraciones podría ser proveniente de herbecidas, insecticidas o plaguicidas que percolan los suelos y perduran en el ambiente (8).

El elemento $\mathrm{Ca}$ aparecen en los cuatro puntos analizados, presentado un máximo en el punto 2 , pero manteniéndose en todos los casos por debajo del límite máximo de $200 \mathrm{mg} / \mathrm{l}$ para aguas naturales (8).

El Hierro también aparece en la totalidad de los puntos muestrales. La menor concentración de este elemento fue para el punto 1, aumentando a partir del punto 2 y manteniéndose prácticamente constante en los puntos 2, 3 y 4 . En todos los puntos las concentraciones de Fe están por encima del valor máximo aceptado para aguas naturales de menor de $1 \mathrm{mg} / \mathrm{l} \mathrm{(8)}$.

La causa de esto podría ser la existencia en las márgenes del río de industrias que utilizan Hierro o sus minerales en procesos de producción y que descargan sus aguas en el río. También existen en las márgenes del río plantas energéticas que utilizan el agua del río como refrigerante y luego la descargan en el mismo, después de circular por tuberías de acero o hierro fundido. Asimismo la disolución de rocas y minerales es causa de la presencia de $\mathrm{Fe}$ en las aguas naturales.

El Zinc, identificado en los cuatro puntos analizados, aparecen en concentraciones que se encuentran dentro del rango considerado normal para agua corriente, $0.01-1 \mathrm{mg} / \mathrm{l}(8)$.

El Níquel, cuya concentración en aguas superficiales debe estar entre 5-20 Ug/l (8), aparece en los puntos 1,2 y 4, presentando un máximo en el punto 2. En todos los puntos las concentraciones de $\mathbf{N i}$ son superiores al límites máximo aceptado. La procedencia de este elemento podría estar en la existencia en barrios ubicados en las márgenes del río, de industrias caseras de reconstrucción de baterías de automóvil y de laminado de metales, así como de industrias que utilizan fungicidas, las cuales probablemente descargan sus desechos en el río.

El Plomo aparece en los puntos 1 y 4 en concentraciones que exceden por mucho los valores límites de este elemento para lagos y ríos. Estos valores son 1-10 Ug/l (8). La presencia de plomo en las 
aguas analizadas, igual que en el caso del nfquel, puede provenir de desechos de la fabricación de baterfas y de talleres de soldadura y pintura ubicada en la orilla del río.

El Arsénico aparece únicamente en el punto 3 en concentraciones superiores al valor aceptado de $10 \mathrm{Ug} / \mathrm{l}(8)$. La mayoría del As presente en el agua proviene de descargas industriales, principalmente de fundiciones y ciertos productos farmacéuticos (8).

El Bromo aparece en los puntos 1 y 2 en concentraciones elevadas, atribuibles a la proximidad del agua del mar. En los puntos 3 y 4 su concentración disminuye drásticamente. No se encontraron parámetros en la bibliografía disponible para el $\mathrm{Br}$ en aguas naturales, por no ser este un elemento habitual en agua no contaminada. En los E.U.A. la concentración mediana de bromoformo $(\mathrm{CHBr})$ para aguas tratadas en $0.005 \mathrm{mg} / \mathrm{l}(8)$. El bromoformo es un producto de la reacción entre el ión Bromo y la materia orgánica naturalmente presente en el agua (8).

El Fósforo, elemento no habitual en aguas naturales no contaminadas aparece en los cuatro puntos muestrales en altas concentraciones. Lo mismo ocurre con el Potasio y el Estroncio. En los casos las concentraciones van disminuyendo al subir rfo arriba, siendo mucho más altas próximo a la desembocadura, cerca del mar. No se encontraron parámetros de referencia para estos tres elementos debido a que su presencia no es habitual en aguas superficiales no contaminadas.

Por ser la técnica TRXRF multielemental, nos ha permitido el análisis simultáneo de elementos con números atómicos comprendido de 19 a 82, algo definitivamente poderoso.

Debido a las altas concentraciones encontradas para cada elemento que con excepción del $\mathrm{Zn}$ y el Ca sobrepasan por mucho los valores máximos permitidos para aguas naturales no contaminadas, puede concluirse que hay alta contaminación química en las aguas del río Ozama, y es muy probable que se deba a descargas industriales en las aguas de dicho río. 


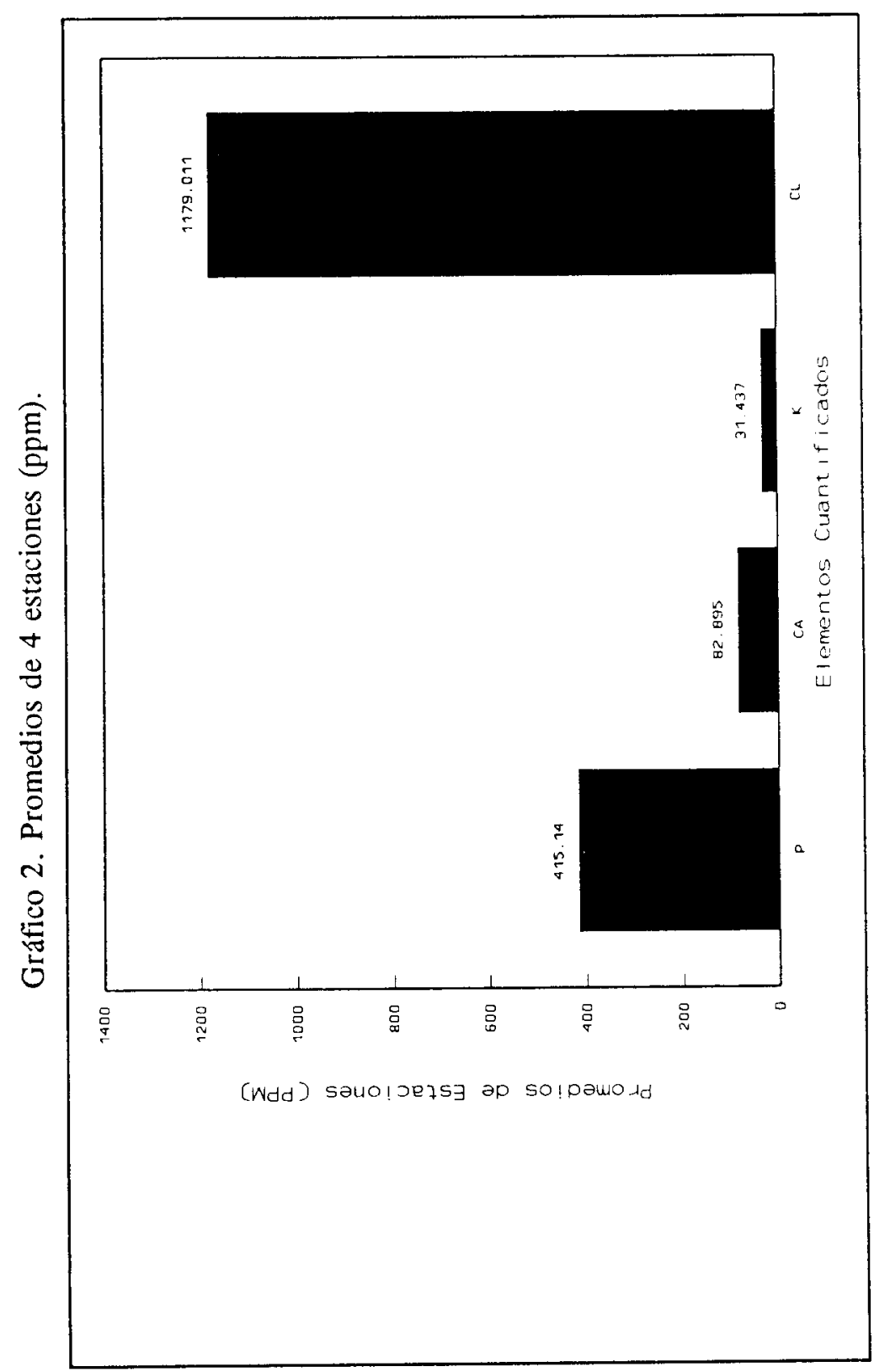




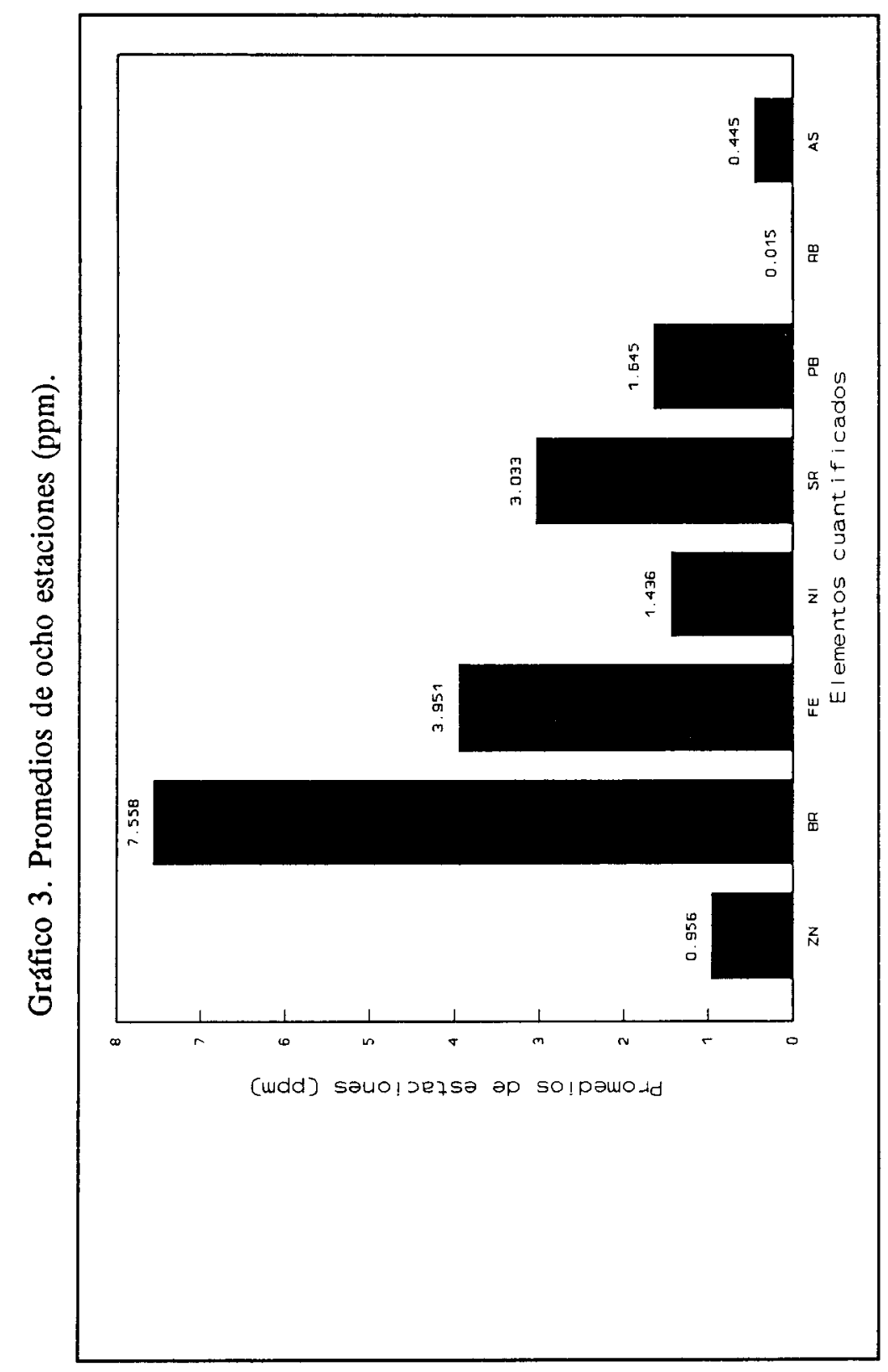

58 


\section{REFERENCIA CITADA}

1. Legden, Donald E. Fundamentals of X-Ray Sepectrometry as applied to energy dispersive techniques. Depto. of Chemistry, Colorado State University, 1984. Tracor X-ray Inc.

2. Pérez E., Oscăr Rafael. Análisis multielemental de $\mathrm{Ca}, \mathrm{Zn}, \mathrm{Cu}, \mathrm{Mn}$, $\mathrm{Ni}, \mathrm{As}, \mathrm{Cr}, \mathrm{Hg}, \mathrm{Pb}, \mathrm{Cl}, \mathrm{S}, \mathrm{K}, \mathrm{V}, \mathrm{Sr}$, Bi, Ti en agua potable de la ciudad de Guatemala, utilizando un sistema de reflexión total de rayos $X$ con un tubo de rayos $\mathrm{X}$ de ánodo de Molibdeno. Universidad de San Carlos de Guatemala, Facultad de Ciencias Químicas y Farmacia, enero 1991.

3. Freimann, Peter. Aplication of TRXRF analysis for the determination of trace metals in the North Sea. Diether Schmidt Spectrochimica acta V44B, N5. 1989, 505-510p.

4. Prawge, Andreas. TRXR Spectrometry: Method and applications. Spectrochimica Acta, V44B, N5. 1989, 437-452p.

5. Halliday, David; Resnick, Robert. Física. Vol. 2, cap. 41, 3era. impresión. diciembre 1971.

6. V. Valkovic-IAEA, viena $1983,19 \mathrm{p}$.

7. Standard Methods for the examination of the water and wastwater. American Public Health Assiciation. Joint Editorial-Board, clesceri, Greenberg, Trussel. Washington 1989.

8. Guía para la calidad del agua potable. Organización Panamericana para la Salud (OPS). Publicación Científica 506. Vol.3, 1987.

\section{AGRADECIMIENTOS}

Este trabajo es parte del informe final sometido a un curso de postgrado en Técnicas Analíticas en la Universidad Autónoma de Santo Domingo, UASD. Agradecemos a nuestros profesores y compañeros su cooperación en el mismo. 